Research Paper

\title{
The Histone H3K9 Demethylase Kdm3b Is Required for Somatic Growth and Female Reproductive Function
}

\author{
Zhaoliang Liu'1,3, Xian Chen ${ }^{1}$, Suoling Zhou ${ }^{1}$, Lan Liao ${ }^{1}$, Rui Jiang ${ }^{1,2}$ and Jianming $\mathrm{Xu}^{1,2,}{ }^{凶}$ \\ 1. Department of Molecular and Cellular Biology, Baylor College of Medicine, Houston, Texas, USA. \\ 2. Luzhou Medical College, Luzhou, Sichuan, China. \\ 3. Institute of Cancer Prevention and Treatment, Harbin Medical University, Harbin, China
}

$\triangle$ Corresponding author: Jianming Xu, One Baylor Plaza, Mail Stop BCM130, Houston, TX 77030; E-mail: jxu@bcm.edul; Phone: 713-798-6199

() 2015 Ivyspring International Publisher. Reproduction is permitted for personal, noncommercial use, provided that the article is in whole, unmodified, and properly cited. See http://ivyspring.com/terms for terms and conditions.

Received: 2015.02.10; Accepted: 2015.03.01; Published: 2015.03.19

\begin{abstract}
$\mathrm{Kdm} 3 \mathrm{~b}$ is a Jumonji $\mathrm{C}$ domain-containing protein that demethylates mono- and di-methylated lysine 9 of histone $\mathrm{H} 3(\mathrm{H} 3 \mathrm{~K} 9 \mathrm{mel}$ and $\mathrm{H} 3 \mathrm{~K} 9 \mathrm{me} 2)$. Although the enzyme activity of $\mathrm{Kdm} 3 \mathrm{~b}$ is well characterized in vitro, its genetic and physiological function remains unknown. Herein, we generated $K d m 3 b$ knockout (Kdm3bKO) mice and observed restricted postnatal growth and female infertility in these mice. We found that $\mathrm{Kdm} 3 \mathrm{~b}$ ablation decreased IGFBP-3 expressed in the kidney by $53 \%$ and significantly reduced IGFBP- 3 in the blood, which caused an accelerated degradation of IGF-1 and a $36 \%$ decrease in circulating IGF-1 concentration. We also found Kdm3b was highly expressed in the female reproductive organs including ovary, oviduct and uterus. Knockout of $\mathrm{Kdm} 3 \mathrm{~b}$ in female mice caused irregular estrous cycles, decreased $45 \%$ of the ovulation capability and $47 \%$ of the fertilization rate, and reduced $44 \%$ of the uterine decidual response, which were accompanied with a more than $50 \%$ decrease in the circulating levels of the 17 beta-estradiol. Importantly, these female reproductive phenotypes were associated with significantly increased levels of $\mathrm{H} 3 \mathrm{~K} 9 \mathrm{mel} / 2 / 3$ in the ovary and uterus. These results demonstrate that Kdm3b-mediated $\mathrm{H} 3 \mathrm{~K} 9$ demethylation plays essential roles in maintenance of the circulating IGF-1, postnatal somatic growth, circulating 17 beta-estradiol, and female reproductive function.
\end{abstract}

Key words: knockout mice, IGFBP-3/IGF-1, ovulation, fertilization, decidulization

\section{Introduction}

The N-terminal tails of histones are subject to posttranslational modifications including methylation, acetylation, ubiquitynation and sumoylation of the lysine residue $(\mathrm{K})$, methylation of the arginine residue, phosphorylation of the serine and threonine residues, and ADP ribosylation of the glutamic acid residue [1]. These modifications play crucial roles in determining chromatin topology, recruitment of nonhistone proteins to chromatin, gene expression profile and genome stability, allowing fine regulation of many fundamental biological processes during development and organ function. Among these various modifications in mammals, monomethylated H3K27,
H3K9, H4K20, H3K79 and H2BK5, as well as the methylated $\mathrm{H} 3 \mathrm{~K} 4$ in the promoter region are usually associated with transcriptional activation [2-4]. High levels of histone $\mathrm{H} 3$ acetylation and $\mathrm{H} 3 \mathrm{~K} 4 \mathrm{me} 1$ are also detected at the functional enhancer regions in different types of cells [5]. On the other hand, high levels of trimethylated H3K27, H3K9 and H3K79 are usually linked with gene repression [4]. Although these histone modifications have been extensively studied at molecular and cellular levels in cell-free biochemical reactions and cultured cells, the physiological functions and pathogenic roles of these histone modifications remain largely unclear. 
Accordingly, many histone modification enzymes have been identified [1]. Among these enzymes, the KDM3 family has three important members, including KDM3A (JMJD1A, JHDM2A or TSGA), KDM3B (JMJD1B, JHDM2B or 5qNCA) and JMJD1C (JHDM2C or TRIP8). These KDM3 family members contain a Jumonji $\mathrm{C}(\mathrm{JmjC})$ domain and serve as histone demethylases. Both KDM3A and KDM3B can interact with androgen receptor (AR) and demethylate H3K9me1/2 [6-8]. Since highly methylated H3K9 is commonly associated with transcriptional repression and formation of heterochromatin, KDM3A/B-mediated demethylation of H3K9 usually enhances transcriptional activation of their target genes [6]. KDM3A can be induced by hypoxia condition to increase the expression of a subset of hypoxia-inducible genes that enhance tumor growth [9]. The $5 q 31$ locus, where the human $K D M 3 B$ gene resides, is deleted in about $1 / 3$ of the myelodysplasia patients. The expression of the JMJD1C gene is reduced in various malignancies [10-12]. Higher KDM3B expression in breast tumors is also associated with a better prognosis [13]. These findings suggest that KDM3B/C may serve as tumor suppressors. However, a recent study revealed that $\mathrm{KDM} 3 \mathrm{~B}$ expression is decreased during differentiation of the acute promyelocytic leukemia cells, suggesting KDM3B may promote oncogenesis of this cancer type [7]. It is currently unknown why KDM3B plays different roles in different types of cancers. In order to understand the pathogenic roles of these KDM3 histone demethylases, it is important to understand their in vivo physiological functions.

$\mathrm{Kdm} 3 \mathrm{a}$ is highly expressed in the testis and adipose tissue [14-16]. Our group and others have previously reported that $K d m 3 a$ knockout male mice had a defective spermatogenesis and were infertile due to impaired gene expression mediated by Crem in germ cells [14, 15]. Kdm3a knockout mice also exhibited obesity because of a decrease in gene expression mediated by Ppar in the adipose tissue [16]. In the present study, we aimed to generate and characterize $K d m 3 b$ knockout (Kdm3bKO) mice in order to define the genetic and physiological function of the $K d m 3 b$ gene. We show that $\mathrm{Kdm} 3 \mathrm{~b}$ is essential for normal somatic growth and female reproductive function.

\section{Materials and Methods}

\section{Targeting the mouse $K d m 3 b$ gene}

The $5^{\prime}$ and $3^{\prime}$ homologous arms of the targeting vector were amplified from the genomic DNA extracted from TC-1 mouse ES cells [17] by PCR using the LA PCR kit (Takara Bio, Otsu, Shiga, Japan) and two pairs of primers, 5F (cggttaattaatggccaa- gatcaaaagatgga) paired with and 5R (aatgcggccgcgtggttctcaggcccattta) and 3F (aatctcgagaaaggactccgggaaactgt) paired with 3R (aaaggatcctagggccacacaggttaagg). The yielded DNA fragments were validated by sequencing and cloned into the pFRT-LoxP targeting vector [18] (Fig. 1A). The targeting vector was linearized and electroporated into TC-1 ES cells with a $129 \mathrm{SvEv} / \mathrm{j}$ strain background. These ES cells were cultured in the selection medium and surviving clones were isolated and screened by Southern blot (Fig. 1A). The targeted ES cell clones were injected into the morulae collected from C57BL/ 6 female mice. The generated male chimeric founders were used to mate with C57BL/ 6 females to produce $K d m 3 b$ heterozygous $\left(K d m 3 b^{+-}\right)$ mice. $\mathrm{Kdm}_{3} \mathrm{~b}^{+-}$mice were further bred to produce WT, $K d m 3 b^{+-}$and $K d m 3 b$ knockout (Kdm3b/- or Dkm3bKO) mice. Mouse genomic DNA was prepared from a small piece of ear skin for genotype analysis as described previously [14]. The WT Kdm3b allele was detected by PCR using Primer 22 (gaagagcaaagccagcctac) and Primer 23 (agagccaggagtgagacgtg). The targeted $\mathrm{Kdm} 3 \mathrm{~b}$ allele was detected by PCR using Primer KOV1 (gaaagtataggaacttcgtcgacctc) and Primer 28 (cacataaacaacactcaagtagcc). Animal protocols were approved by the Institutional Animal Care and Use Committee at Baylor College of Medicine.

\section{Quantitative RT-PCR}

RNA samples were isolated from mouse tissues using the Trizol Reagent (Life Technology, Grand Island, NY). Gene-specific primer pairs and matched universal mouse probe sets (Roche Applied Science, Indianapolis, IN) were used to measure mRNA concentrations relative to the endogenous 18S RNA concentrations.

\section{IGF-binding protein (IGFBP) assay}

IGFBPs were assayed as described previously [19]. Briefly, proteins in $0.5 \mu \mathrm{l}$ of serum from 12 week-old mouse were separated in SDS-PAGE gel and blotted onto a nitrocellulose membrane. After blocking and washing, the membrane was incubated overnight at $4^{\circ} \mathrm{C}$ with $5 \times 10^{5} \mathrm{cpm}$ of human ${ }^{125}$ I-IGF-1 (hIGF-1) (Perkin Elmer Life and Analytical Sciences, Waltham, MA). The membrane was washed with $0.1 \%$ Tween-20 in saline and exposed to X-ray film for 2 days.

\section{In vivo hIGF-1 degradation assay}

Fifty microliter blood was collected from the tail tip of each 12-week-old male WT or Kdm3bKO mouse as baseline samples. hIGF-1 was obtained from the National Hormone \& Pituitary Program (Harbor-UCLA Medical Center, Torrance, CA). hIGF-1 diluted in PBS was intraperitoneally injected into WT 
and $\mathrm{Kdm} 3 \mathrm{~b}^{-/}$mice at a dose of $0.25 \mu \mathrm{g} / \mathrm{g}$ body weight. About $50 \mu \mathrm{l}$ of blood was collected from each mouse at $0.333,3.333,5$ and 20 hours after hFGF-I was injected. hIGF-1 concentrations were measured using the hIGF-1-specific ELISA kit (DSL-5600, Diagnostic Systems Laboratories, Webster, TX).

\section{Western blot}

Protein samples were prepared from mouse tissues and protein concentrations were measured as described previously [14]. Western blot analysis was also performed as described previously [14].

\section{Histological examination and immunohisto- chemistry (IHC)}

Tissues were fixed in $4 \%$ paraformaldehyde in PBS and embedded in paraffin. Tissue sections were cut at the thickness of 5 micron and subjected to H\&E staining or IHC as described previously [14].

\section{Antibodies}

This study used antibodies against $\mathrm{Kdm} 3 \mathrm{~b}$ (2621S) from Cell Signaling (Boston, MA), histone H3 (ab1791) and histone H3K9me1 (ab9045) from Abcam (Cambridge, MA), histone H3K9me2 (07-441) and histone H3K9me3 (07-442) from Millipore (Billerica, $\mathrm{MA}$ ), and $\beta$-actin (A2228, Clone ac-74) from Sigma-Aldrich (St. Louis, MO).

\section{Examination of ovulation and fertilization}

Six-week-old female WT and Kdm3bKO mice were housed with adult WT males. The morning when the coital plug was observed was recorded as dpc 0.5. Mice with coital plugs were sacrificed at dpc 1.5 for collecting oocytes as described previously [20]. Total number of oocytes and the number of fertilized oocytes at 2-cell stage were counted under a stereomicroscope.

To test the superovulation capability, adult mice were intraperitoneally injected with 5 IUs of pregnant mare serum gonadotropin (PMSG) (EMD Chemicals Inc.) in the afternoon. Forty-six hours later, mice were intraperitoneally injected with 5 IUs of human chorionic gonadtropin (hCG) (Novarel) and then individually housed with a wild type male mouse. The female mice were sacrificed next day, and their oviducts were collected in M2 medium. The oocytes with their surrounding cumulus cells were released from the ampulla by using a pair of forceps with sharp tips. Oocytes were separated from cumulus cells by hyaluronidase digestion for 2 minutes and pipetting up and down several times. The oocytes were washed in M2 medium for 3 times and then transferred to the droplets of M2 medium (20 $\mu \mathrm{l} /$ drop, 10-15 oocytes/drop) covered with light mineral oil in a plastic Petri dish. The oocytes were cultured at $37^{\circ} \mathrm{C}$ in an incubator supplied with $5 \% \mathrm{CO}_{2}, 5 \% \mathrm{O}_{2}$ and $90 \% \mathrm{~N}_{2}$ for one day. The number of total oocytes and 2-cell stage embryos were counted and recorded.

\section{Examination of the implantation sites}

Age-matched adult female WT and $\mathrm{Kdm} 3 \mathrm{bKO}$ mice were mated with adult WT males. On dpc 5, 100 $\mu l$ of Chicago Blue (1\% solution) were injected into the tail vein of each anaesthetized pregnant mouse. Three minutes later, the injected mouse was sacrificed and their uteri were dissected out for examining the blue implantation sites.

\section{Decidual response assay}

Age-matched female WT and Kdm3bKO mice were ovariectomized and allowed to rest for 2 weeks. These mice were sequentially treated with $17 \beta$-estradiol for three days, and then with $17 \beta$-estradiol and progesterone for five days after a two-day interval as described previously [20]. Six hours after the third day $17 \beta$-estradiol and progesterone injection, the inner wall of one uterine horn in each mouse was mechanically scratched using a bended 18G needle as described previously [21]. The other un-scratched uterine horn served as a control. Six hours after the last injection of hormones, these mice were sacrificed and their traumatized and control uterine horns were dissected out and weighed. The collected tissues were used for preparing frozen sections for histochemical analysis of the alkaline phosphotase activity as described previously [20].

\section{Measurement of hormones}

Serum was prepared from 8-12 week old WT and $\mathrm{Kdm} 3 \mathrm{bKO}$ mice. IGF-1 concentration in the serum was assayed using a Mouse/Rat IGF-1 ELISA kit (DSL-10-29200, Diagnostic Systems Laboratories, Webster, TX). The concentrations of the $17 \beta$-estradiol and progesterone in the serum were measured using the RIA kits DSL-4400 and DSL-3900, respectively (Diagnostic Systems Laboratories, Webster, TX).

\section{Analysis of uterine responses to estrogen and progesterone treatments}

Female adult WT and Kdm3bKO mice were treated with $17 \beta$-estradiol and progesterone as described previously to determine the uterine responsiveness to steroid stimuli [22]. Briefly, mice were ovariectomized, allowed to rest for 2 weeks, and then treated with vehicle (sesame oil, s.c.), $17 \beta$-estradiol (100 ng/mouse/day, s.c.), or $17 \beta$-estradiol (100 $\mathrm{ng} /$ mouse/day) plus progesterone (1 $\mathrm{mg} / \mathrm{mouse} /$ day) for 3 days. Six hours after the final treatment, mice were sacrificed, and their uteri were weighted and normalized to their body weights. 


\section{Results}

\section{Generation of $K d m 3 b$ knockout mice}

The mouse $K d m 3 b$ gene contains 24 exons and spans $84 \mathrm{~kb}$ in length. We constructed a targeting vector for deleting its exons 15-23 (Fig. 1A). Since this deletion region codes the JmjC domain essential for the demethylase activity of $\mathrm{Kdm} 3 \mathrm{~b}$, the targeted allele should completely lose its demethylase activity. From screening a total of 600 individual ES colonies by Southern blot, we identified two targeted clones by both $5^{\prime}$ and $3^{\prime}$ probes (Fig. $1 \mathrm{~A}$ and B). Five male chimeric mice were generated by microinjecting these targeted ES cells into the mouse blastocysts and transferring these blastocysts into the uteri of foster mothers. WT, $\mathrm{Kdm}_{3} \mathrm{~b}^{+-}$and $\mathrm{Kdm} 3 \mathrm{bKO}$ mice were subsequently generated from breeding these male chimeric mice with WT C57BL/6 female mice (Fig. 1C).

Western blot analysis of the liver and other tissues validated the absence of $\mathrm{Kdm} 3 \mathrm{~b}$ protein in
Kdm3bKO (Fig.1D and data not shown). Kdm3b+/mice showed no phenotypical difference from WT mice. However, Kdm3bKO mice were smaller and lesser active than their WT littermates at weaning time. Among 160-recorded newborn pups derived from $\mathrm{Kdm}_{3} \mathrm{~b}^{+-}$parents, there were $46 \mathrm{WT}, 83 \mathrm{Kdm} 3 \mathrm{~b}^{+/}$ and $31 \mathrm{Kdm} 3 \mathrm{bKO}$ pups. The number of newborn $\mathrm{Kdm} 3 \mathrm{bKO}$ pups was not significantly different from the number of newborn WT pups $\left(\chi^{2}=3.7, \mathrm{p}>0.05\right)$. $\mathrm{Kdm} 3 \mathrm{bKO}$ mice exhibited frequent postnatal death before weaning. Among the 31 closely monitored Kdm3bKO pups, 11 died within 48 hours after birth and the remaining 20 survived to adulthood. Among a total of 1643 mice produced from $\mathrm{Kdm} \mathrm{b}^{+/}$mating pairs and genotyped at postnatal day 26 (P26), we identified $498 \mathrm{WT}, 872 \mathrm{Kdm} 3 b^{+-}$and $273 \mathrm{Kdm} 3 \mathrm{bKO}$ mice. The number of $\mathrm{Kdm} 3 \mathrm{bKO}$ mice at this stage became significantly lesser than the predicted number 457 according to Mendelian Genetics $\left(\chi^{2}=79.6\right.$, $p<$ $0.0005)$, suggesting that the $K d m 3 b$ gene function is required for normal survival before weaning.
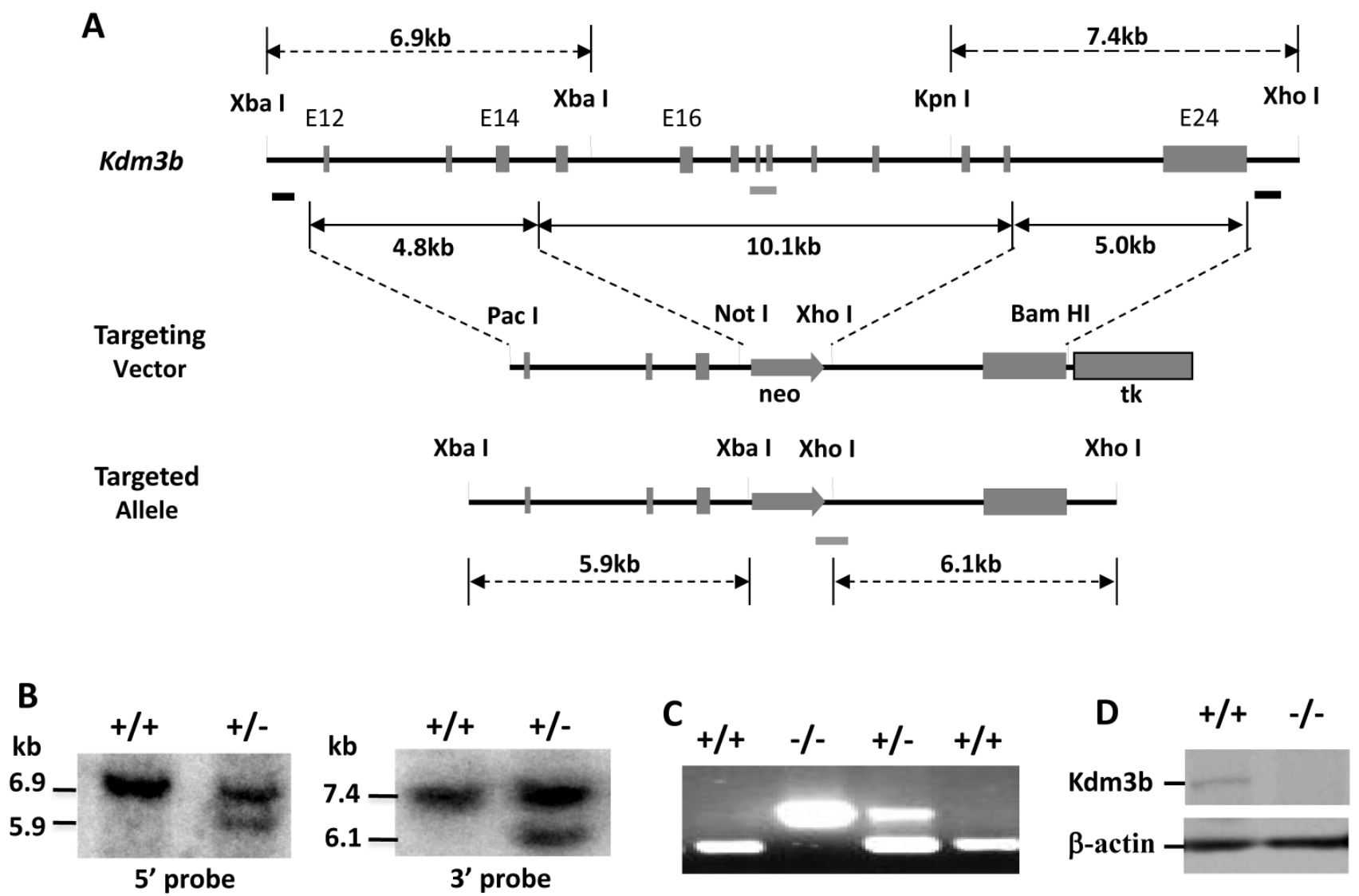

Figure 1. Generation of $K d m 3 b$ knockout mice. $(\boldsymbol{A})$. The gene targeting strategy. The $K d m 3 b$ genomic locus, targeting vector and targeted allele are sketched. The two black bars under the Kdm3b locus indicate the probes used in Southern blot analysis. The grey bars under the gene locus and the targeted allele indicate the DNA fragments amplified by PCR in genotype analysis. (B). Identification of correctly targeted ES clones by Southern blot. Representative results from a WT (+/+) clone and a heterozygously targeted (+/-) clone are presented. The $6.9 \mathrm{~kb}$ and $5.9 \mathrm{~kb}$ bands detected by the 5 ' probe and the $7.4 \mathrm{~kb}$ and $6.1 \mathrm{~kb}$ bands detected by the 3 ' probe represent the WT and the targeted alleles as indicated. (C). PCR-based genotype analysis of WT (+/+), heterozygous (+/-) and knockout (-/-) Kdm3b mice. The upper 300 bp and the lower 179 bp DNA fragments were amplified by PCR using allele-specific primer pairs from the knockout and the WT alleles, respectively. (D). Western blot analysis of Kdm3b protein in the liver lysates of WT (+/+) and $\mathrm{Kdm} 3 \mathrm{bKO}(-/-)$ mice. Beta-actin serves as a loading control. 
A

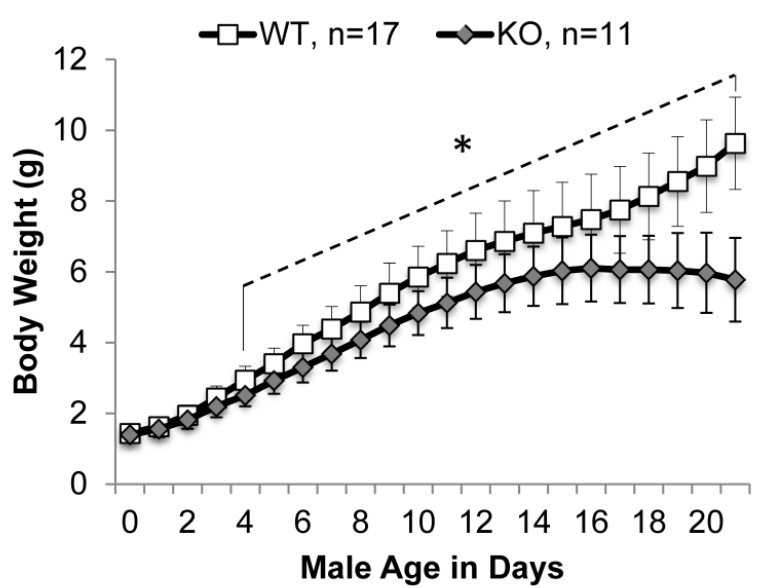

B

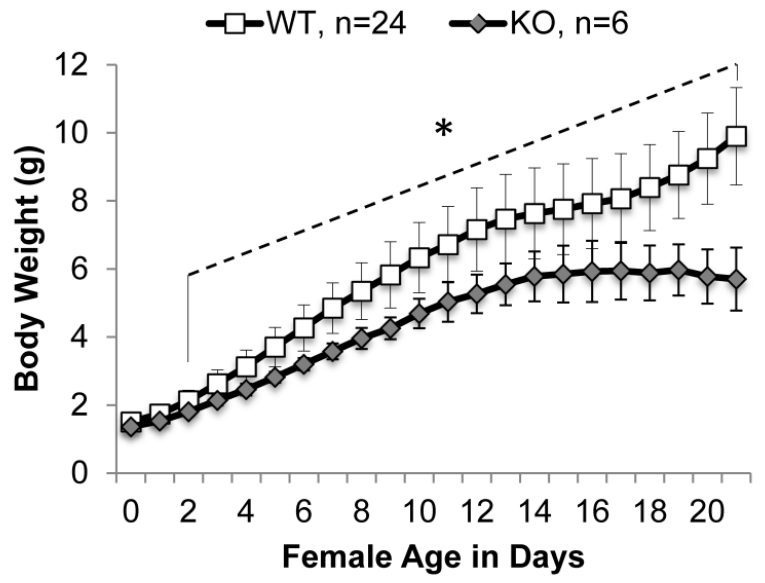

C

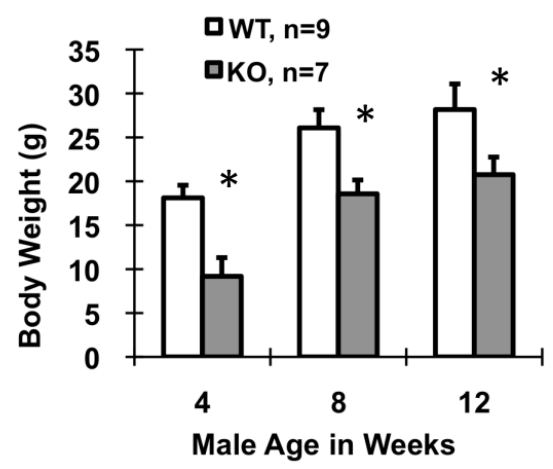

D

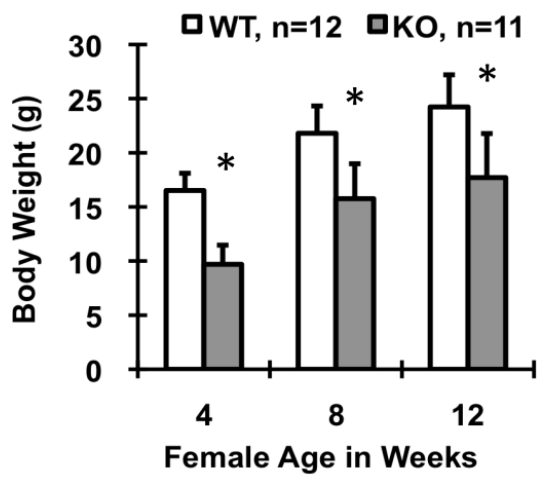

Figure 2. Ablation of $K d m 3 b$ in mice stunted postnatal somatic growth. (A) and (B). Postnatal growth curves of male and female WT and Kdm3bKO mice from P0 to P21. The "*” indicates $p<0.05$ by Student's $t$ test for all age points from postnatal day 4 to day 21 (Panel A) or from postnatal day 2 to day 21 (Panel B). (C) and (D). Body weight comparison between male and female WT and Kdm3bKO mice at ages of 4, 8 and 12 weeks as indicated. The genotype and animal number for each group are indicated in each panel. The "*” in Panels C and D indicates P $<0.05$ by Student's t test.

\section{Kdm3bKO mice exhibited restricted somatic growth, low levels of circulating IGF-1 and IGFBP-3 and fast degradation of IGF-I}

Male and female newborn Kdm3bKO pups had comparable body weights to their WT littermates, but became significantly smaller starting on P4 and P2, respectively (Fig. 2A and B). Their small body size was maintained at a significant level through their lifespan (Fig. 2C and D). In agreement with their smaller body size, the average IGF-1 concentration in $\mathrm{Kdm} 3 \mathrm{bKO}$ mouse sera was reduced $36 \%$ when compared with age-matched WT mouse sera (Fig. 3A). However, IGF-1 mRNA levels in the liver of $\mathrm{Kdm} 3 \mathrm{bKO}$ mice were not significantly different from that in WT mice (Fig. 3B), suggesting that $\mathrm{Kdm} 3 \mathrm{~b}$ deficiency does not affect the growth hormone (GH)-regulated transcription of the IGF-1 gene and the low IGF-1 concentration in Kdm3bKO mouse sera may be caused by its fast degradation. To measure the degradation speed of IGF-1 in vivo, we injected an equal dose of human IGF-1 (hIGF-1) into WT and
Kdm3bKO mice and monitored its change in the serum over a time course. At time 0 (before injection), no hIGF-1 was detected in both WT and Kdm3bKO mouse sera, indicating that the assay had no cross-reaction with the endogenous mouse IGF-1. In 20 minutes, hIGF-1 reached about $300 \mathrm{ng} / \mathrm{ml}$ in both $\mathrm{WT}$ and $\mathrm{Kdm} 3 \mathrm{~b}^{--}$mice, indicating that the intraperitoneally injected hIGF-1 was equally absorbed by both groups of mice. At the time points of 200 and 300 minutes, the serum hIGF-1 concentration was reduced $50 \%$ and $73 \%$ in WT mice, while it was reduced as much as $73 \%$ and $90 \%$ in $\mathrm{Kdm} 3 \mathrm{bKO}$ mice when compared with that at the 20-minute time point. Statistical analysis indicated that hIGF-1 in Kdm3bKO mice was significantly lower than that in WT mice at the time point of 300 minutes. At the time point of 20 hours, the injected hIGF-1 was completely degraded in both types of mice (Fig. 3C). These results demonstrate that the circulating IGF-1 has a much shorter half-life in $\mathrm{Kdm} 3 \mathrm{bKO}$ versus WT mice, which may partially explain the low IGF-1 concentrations and restricted somatic growth of $\mathrm{Kdm} 3 \mathrm{bKO}$ mice. 
C
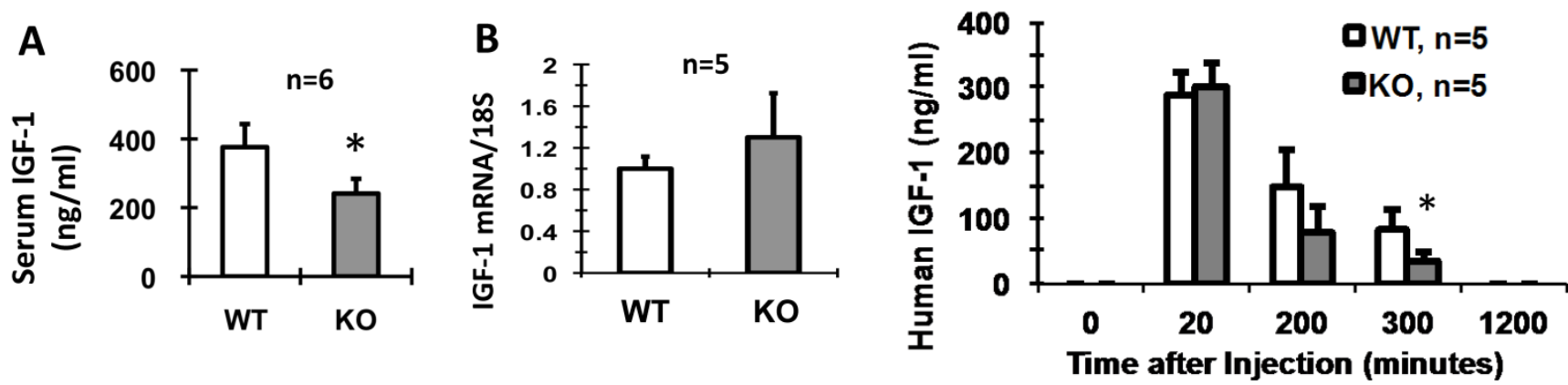

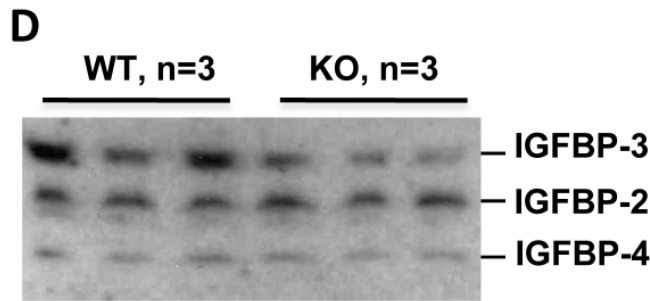

$\mathbf{E}$

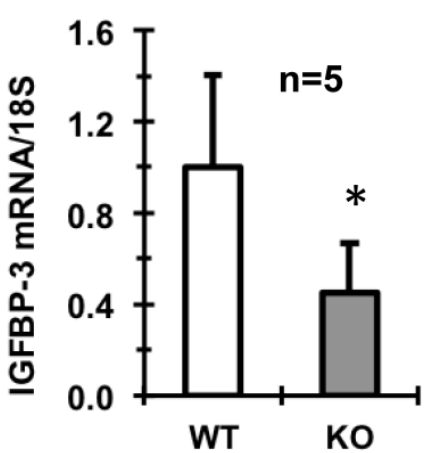

$\mathbf{F}$

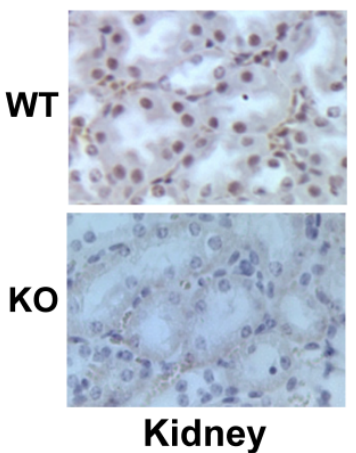

Figure 3. Kdm3bKO mice exhibit lower serum IGF-1 without changing IGF-I mRNA expression in the liver (Panels A and B), faster IGF-1 degradation in the blood circulation (Panel C), lower serum IGFBP-3 (Panel D), and decreased IGFBP-3 mRNA expression in the kidney (Panel E). All animals used in these experiments were 8-10 weeks old male mice. Kdm3b protein is detected by IHC in the kidney epithelial cells of WT mice but not in the kidney of Kdm3bKO mice (Panel F). The number of mice in each genotype group is indicated in each panel. In Panels B and E,the relative expression levels of IGF-1 and IGFBP-3 mRNAs were measured by qPCR. In Panel C, a human IGF-1 specific antibody in an ELISA kit was used to specifically measure the injected human IGF-1 at each time point. In Panel D, IGFBPs were measured by ligand blotting using ${ }^{125}$ I-IGF-1. *, P < 0.05 by Student's t test.

Since IGF-1-binding proteins (IGFBPs) play crucial roles in stabilizing IGF-1 [19], we compared their levels in the sera of $\mathrm{WT}$ and $\mathrm{Kdm} 3 \mathrm{bKO}$ mice. We found that IGFBP-2 and IGFBP-4 levels were comparable, but the IGFBP-3 levels were much lower in $\mathrm{Kdm} 3 \mathrm{bKO}$ mice versus WT mice (Fig. 3D). Furthermore, the IGFBP-3 mRNA level in the kidney, the organ expressing high IGFBP-3 mRNA [19], was also significantly lower in $\mathrm{Kdm} 3 \mathrm{bKO}$ mice versus WT mice (Fig. 3E). Immunohistochemistry (IHC) analysis clearly detected $\mathrm{Kdm} 3 \mathrm{~b}$ protein present in the epithelial nuclei of the kidney tubules and Bowman's capsules of WT mice, but absent in the Kdm3bKO mouse kidneys (Fig. 3F). Taken together, these results suggest that knockout of $K d m 3 b$ decreases IGFBP-3 expression and its circulating level, which in turn causes IGF-1 destabilization in Kdm3bKO mice.

\section{The female Kdm3bKO mice exhibited a se- verely impaired reproductive function}

To examine the reproductive function of $\mathrm{Kdm} 3 \mathrm{bKO}$ mice, we carried out a 4-month long breeding experiment and summarized the data in Table 1. The five WT breeding pairs produced a total of 148 pups. However, the four breeding pairs of female Kdm3bKO and male WT mice did not produce any pups in this experiment. From all other breeding pairs in the entire study, we only observed one Kdm3bKO female mouse delivered three pups, but these pups died shortly right after birth. These results indicate that the female $\mathrm{Kdm} 3 \mathrm{bKO}$ mice are largely infertile.

Table 1. Kdm3bKO mice exhibited a compromised reproductive function.

\begin{tabular}{lllllll}
\hline Mating Setup & $\begin{array}{l}\text { Mat- } \\
\text { ing } \\
\text { Pairs }\end{array}$ & $\begin{array}{l}\text { Total } \\
\text { Pups }\end{array}$ & $\begin{array}{l}\text { Pups/Pair Total } \\
\text { Litters }\end{array}$ & Litters/Pair & Pups/Litter \\
& & & & \\
\hline 우WT X ठWT & 5 & 148 & $29.6 \pm 9.4$ & 18 & $3.6 \pm 1.1$ & $8.2 \pm 2.6$ \\
우KO X ○WT & 4 & 0 & $0^{*}$ & 0 & $0^{*}$ & $0^{*}$ \\
\hline
\end{tabular}

Eight-week-old female and male mice with the indicated genotypes were housed together at 1:1 ratio for four months. Pups derived from each breeding pair were recorded. ${ }^{*}, \mathrm{P}<0.05$ by Student's $\mathrm{t}$ test. 


\section{Knockout of $K d m 3 b$ increased the levels of H3K9mel, H3K9me2 and H3K9me3 in the ovary and uterus}

As the first step to understand the physiological functions of $\mathrm{Kdm} 3 \mathrm{~b}$ in female reproductive organs, we examined its expression profiles in these organs. Western blot analysis detected high levels of the 200 $\mathrm{kD} \mathrm{Kdm3b}$ in the ovary and uterus of WT mice (Fig. $4 \mathrm{~A})$. In the ovary, IHC identified high levels of $\mathrm{Kdm} 3 \mathrm{~b}$ in the nuclei of granulosa cells of the primary, preantral and antral follicles and in the nuclei of thecal cells of the preantral and antral follicles. A weaker immunoreactivity of $\mathrm{Kdm} 3 \mathrm{~b}$ was also detectable in the nuclei of corpus luteum and interstitial stroma cells (Fig. 4B and data not shown). In the oviduct, $\mathrm{Kdm} 3 \mathrm{~b}$ was mainly detected in the nuclei of epithelial cells (Fig. 4C). In the uterus, Kdm3b was mainly detected in the nuclei of endometrial luminal and glandular epithelial cells and myometrial smooth muscle cells. Slightly weaker signals of $\mathrm{Kdm} 3 \mathrm{~b}$ immunostaining were also detected in the nuclei of certain stromal cells (Fig. 4D). No Kdm3b was detected in the ovary, oviduct and uterus of $\mathrm{Kdm} 3 \mathrm{bKO}$ mice as expected (Fig. 4B-D). These results indicate that $\mathrm{Kdm} 3 \mathrm{~b}$ is highly and extensively expressed in the ovary, oviduct and uterus and hence may play important roles in modifying the epigenetic histone codes in these organs.
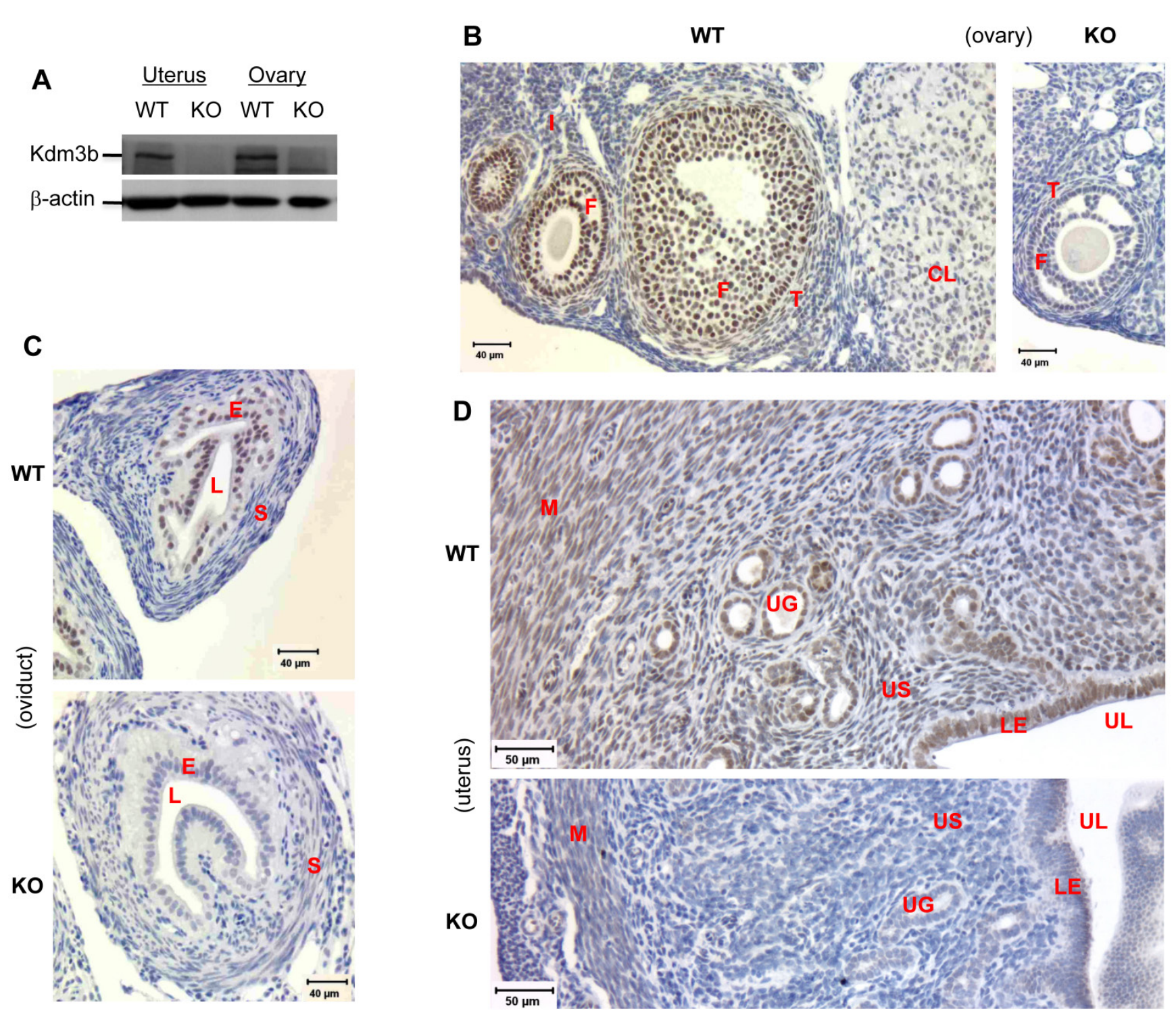

Figure 4. $\mathrm{Kdm} 3 \mathrm{~b}$ is highly expressed in the female mouse reproductive tracts. (A). Western blot analysis of $\mathrm{Kdm} 3 \mathrm{~b}$ expression in the uteri and ovaries of WT and $\mathrm{Kdm} 3 \mathrm{bKO}(\mathrm{KO})$ mice. (B). IHC analysis of Kdm3b protein in the ovary of WT mice. The ovarian section of Kdm3bKO mice served as a negative control of IHC. I, interstitial stroma; $F$, follicles; T, thecal cells; $C L$, corpus luteum. (C). IHC analysis of Kdm3b in the oviduct. The oviduct section of $\mathrm{KO}$ mice served as a negative control of IHC. E, epithelium; S, stroma; L, lumen. (D). IHC analysis of Kdm3b in the uteri of WT mice, and Kdm3bKO mice (negative control of IHC). M, myometrium; UG, uterine gland; US, uterine stroma; LE, luminal epithelium; UL, uterine lumen. In the images of all IHC analysis, the Kdm3b immunostaining signal is in brown, and the cell nuclei stained by hematoxylin are in blue. 


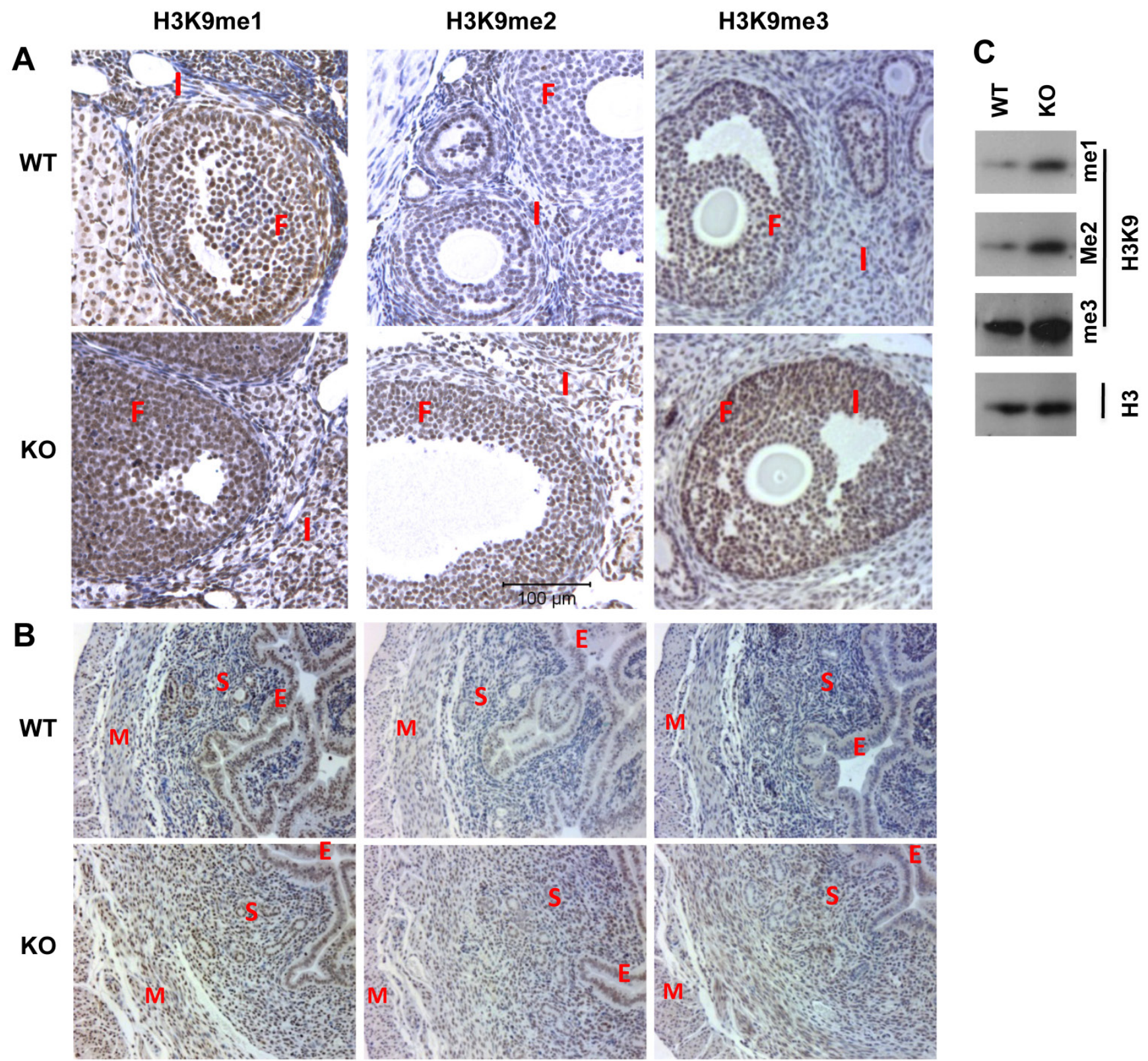

Figure 5. Knockout of $K d m 3 b$ increases the levels of $\mathbf{H} 3 \mathrm{~K} 9$ methylation in the ovary and uterus. (A) $\&$ (B). $I H C$ analyses of $H 3 K 9 m e 1 / 2 / 3$ in the ovaries and uteri of WT and Kdm3bKO (KO) mice. F, follicular cells; I, interstitial cells; E, endometrial epithelium; S, endometrial stroma; M, myometrium. Two mice and multiple sections from each mouse in each genotype group were examined. (C). Analysis of $\mathrm{H} 3 \mathrm{~K} 9 \mathrm{me} 1 / 2 / 3$ in the uteri of WT and $\mathrm{KO}$ mice by Western blotting. Histone $\mathrm{H} 3$ served as a loading control.

In the ovaries of WT mice with synchronized estrous cycle induced by PMSG and hCG treatments, relatively high levels of $\mathrm{H} 3 \mathrm{~K} 9 \mathrm{me} 1$ were detected in the follicular granulosa, interstitial stromal cells and corpus luteum. Knockout of $\mathrm{Kdm} 3 \mathrm{~b}$ further increased the signals of $\mathrm{H} 3 \mathrm{~K} 9 \mathrm{me} 1$ immunostaining in these cells. In WT ovaries, low levels of H3K9me2 were observed in the follicular granulosa and interstitial stromal cells. However, in the ovaries isolated from $\mathrm{Kdm} 3 \mathrm{bKO}$ mice, $\mathrm{H} 3 \mathrm{~K} 9 \mathrm{me} 2$ levels were increased in both of granulosa and interstitial stromal cells. H3K9me3 levels were detected at medium levels in WT granulosa cells, but at high levels in $\mathrm{Kdm} 3 \mathrm{bKO}$ granulosa cells. On the other hand, H3K9me3 levels were similar in both WT and $\mathrm{Kdm} 3 \mathrm{bKO}$ interstitial stromal cells (Fig. 5A and data not shown). In WT uteri, H3K9me1 immunoreactivity was present in the nuclei of luminal and glandular epithelial cells, some endometrial stromal and myometrial smooth muscle cells. H3K9me2 was mainly detected in the luminal epithelial cells, some endometrial stromal cells and most myometrial smooth muscle cells, but only a few glandular epithelial cells were H3K9me2 positive. H3K9me3 immunoreactivity was weak and only presented in small numbers of luminal epithelial, endometrial stromal and myometrial smooth muscle cells 
(Fig. 5B and data not shown). Importantly, in $\mathrm{Kdm} 3 \mathrm{bKO}$ uteri, H3K9me1 was elevated in the endometrial stromal and myometrial smooth muscle cells, H3K9me2 was induced in all glandular epithelial and most endometrial stromal cells, and H3K9me3 was increased in nearly all uterine cells, especially in the glandular epithelial cells (Fig. 5B and data not shown). In agreement with these IHC results, Western blot analysis also demonstrated that the levels of H3K9me1, H3K9me2 and H3K9me3 were significantly increased in $\mathrm{Kdm} 3 \mathrm{bKO}$ uteri versus WT uteri (Fig. 5C). Taken together, these results indicate that $\mathrm{Kdm} 3 \mathrm{~b}$ is required for maintaining normal patterns of H3K9 methylation in multiple types of cells in the female reproductive organs. Kdm3b deficiency altered these epigenetic codes and disrupted the female reproductive function.

\section{Knockout of $K d m 3 b$ in female mice prolonged their estrous cycles and reduced their ovula- tion capacity and fertilization efficiency}

To explore the pathophysiological causes for the impaired reproductive function of the female $\mathrm{Kdm} 3 \mathrm{bKO}$ mice, we examined their sexual maturation by checking vaginal opening time and their estrous cycles, which are regulated by estrogen and progesterone produced from the ovarian thecal and corpus luteum cells expressing $\mathrm{Kdm} 3 \mathrm{~b}$. $\mathrm{Kdm} 3 \mathrm{bKO}$ and WT mice showed similar vaginal opening ages from P28 to P40 (Supplementary Material: Fig. S1A), indicating that $\mathrm{Kdm} 3 \mathrm{~b}$ is not required for female sexual maturation. The adult female WT mice $(n=5)$ exhibited normal estrous cycles with 4-5 days per cycle. However, only 2 of 6 examined female Kdm3bKO mice showed normal estrous cycles, another three showed prolonged estrous cycles and the remaining one stayed at the diestrus phase for 12 days (Supplementary Material: Fig. S1B and data not shown). These results suggest that $\mathrm{Kdm} 3 \mathrm{~b}$ function is involved in maintaining normal estrous cycle.

Next, we compared the morphology and function of Kdm3bKO and WT ovaries. We observed that all stages of follicles were present and corpus luteums formed normally in Kdm3bKO ovaries (Supplementary Material: Fig. S2A). We further compared ovulation function and fertilization efficiency in $\mathrm{Kdm} 3 \mathrm{bKO}$ and WT female mice at dpc 1.5. Five WT females discharged an average of 8.4 oocytes per mouse, while 4 of 5 examined $\mathrm{Kdm} 3 \mathrm{bKO}$ females only discharged an average of 4.6 oocytes per mouse. Interestingly, about $81 \%$ (34 out of 42 ) of oocytes in WT females were fertilized, but only $43 \%$ (10 out of 23 ) of oocytes in $\mathrm{Kdm} 3 \mathrm{bKO}$ females were fertilized (Fig. 6A and B). The fertilized oocytes harvested from Kdm $3 b K O$ mice also exhibited abnormal morphologies when com- pared with those from WT mice (Fig. 6A). Furthermore, although PMSG and hCG treatment-induced super ovulation was not significantly different between $\mathrm{Kdm} 3 \mathrm{bKO}$ and WT mice, the fertilization rate in $\mathrm{Kdm} 3 \mathrm{bKO}$ mice was only $8 \%$, which was almost 7 fold lower than the $54 \%$ fertilization rate in WT mice (Fig. $6 \mathrm{C}$ and D). These results suggest that $\mathrm{Kdm} 3 \mathrm{~b}$ is required for producing normal number of oocytes with high quality and for maintaining an efficient fertilization rate.

\section{Female Kdm3bKO mice had a reduced num- ber of embryos and a decreased uterine de- cidual response}

In agreement with reduced ovulation and fertilization, the pregnant rate of $\mathrm{Kdm} 3 \mathrm{bKO}$ mice was also lower than that of WT mice after successful coitus as examined on dpc 4.5 and dpc 7.5. For the same reason, pregnant Kdm3bKO mice also carried fewer embryos than pregnant WT mice did on dpc 4.5 and dpc 7.5 (Supplementary Material: Table S1). The embryonic implantation sites in $\mathrm{Kdm} 3 \mathrm{bKO}$ uteri were fewer and smaller when compared with those in WT uteri (Fig. 7A and B, and Table S1). Most embryos in Kdm3bKO uteri were either small or were being absorbed (Fig. 7C and data not shown). The small implantation sites and the failure in maintaining embryo growth and survival at these pregnant stages suggest that the endometrial stroma of $\mathrm{Kdm} 3 \mathrm{bKO}$ mice might have an abnormal decidualization.

Therefore, we assayed decidual responses in adult WT and Kdm3bKO mice. In this widely used assay, estrogen and progesterone-promoted decidual response was induced by a mechanical scratch on the internal wall of one uterine horn (Fig. 7D). This estrogen-primed, progesterone-dependent, and mechanical trauma-induced decidualization was robust in WT uteri, as the average weight of their scratched uterine horns was 2.7 fold of that of their un-scratched horns. However, the decidual response of $\mathrm{Kdm} 3 \mathrm{bKO}$ uteri was limited, as the average weight of their scratched uterine horns was only 1.5 fold of that of their un-scratched horns (Fig. 7D). Accordingly, the alkaline phosphotase activity, a hallmark of decidual response, was induced at much lower degrees in the scratched uterine horns of $\mathrm{Kdm} 3 \mathrm{bKO}$ mice versus those of WT mice (Fig. 7E). These results indicate that $\mathrm{Kdm} 3 \mathrm{~b}$ is required for normal uterine decidualization.

\section{Kdm3bKO female mice exhibited decreased levels of estradiol}

The functions of the female reproductive organs are tightly regulated by ovarian steroid hormones including estradiol and progesterone. After dpc 4.5, a high level of progesterone and a low level of estradiol 
in mice work coordinately to maintain normal pregnancy [23]. On dpc 7.5, the average concentration of $17 \beta$-estradiol in the sera of $\mathrm{Kdm} 3 \mathrm{bKO}$ female mice was only $50 \%$ of that in the sera of WT female mice. In WT and Kdm3bKO female mice with estrous cycles synchronized by PMSG and hCG treatments, the levels of $17 \beta$-estrodiol were also significantly lower in the sera of Kdm3bKO mice versus WT mice at 40 hours post PMSG treatment and at 24 hours post hCG treatment (Fig. 8A). However, the progesterone concentration showed no significant difference between these two mouse groups (Fig. 8B). Since the majority of $17 \beta$-estradiol is synthesized by the aromatase (Cyp19a1) expressed in the ovarian granulosa cells where $\mathrm{Kdm} 3 \mathrm{~b}$ is highly expressed, we examined Cyp19a1 expression in the ovaries of Kdm3bKO mice. Real-time RT-PCR analysis revealed no difference in Cyp19a1 mRNA expression between Kdm3bKO and WT ovaries (Fig. 8C), suggesting that Kdm3b knock- out-induced decrease in $17 \beta$-estradiol is not caused by any changes of Cyp19a1 expression.

Finally, we observed that the uteri in sexually matured WT and Kdm3bKO mice showed comparable histological morphology (Supplementary Material: Fig. S2B), suggesting that $\mathrm{Kdm} 3 \mathrm{~b}$ is not required for uterine morphogenesis in the virgin mice. Furthermore, $17 \beta$-estradiol treatment stimulated comparable and robust growth of uteri in both ovariectomized WT and Kdm3bKO mice, indicating that $\mathrm{Kdm} 3 \mathrm{~b}$ is not essential for $17 \beta$-estradiol-induced uterine growth. Moreover, a combined treatment of $17 \beta$-estradiol and progesterone also equally blunted the uterine growth stimulated by the $17 \beta$-estradiol alone in both ovariectomized WT and Kdm3bKO mice (Supplementary Material: Fig. S2C and D), suggesting that $\mathrm{Kdm} 3 \mathrm{~b}$ is also not essential for progesterone signaling in the uterus.
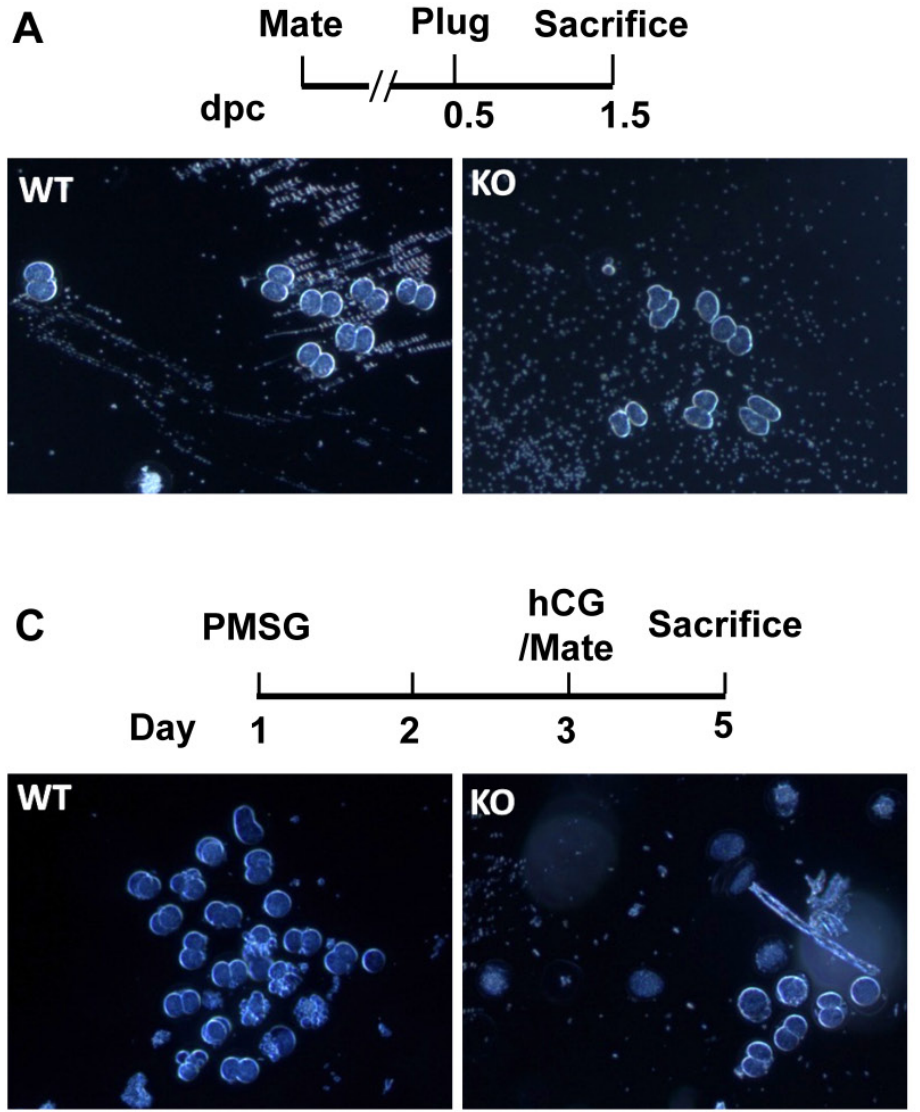

Figure 6. Effects of $\mathbf{K d m} \mathbf{3 b}$ knockout on ovulation and fertilization. (A). Representative images of oocytes ovulated by WT and Kdm3bKO (KO) mice on dpc 1.5. Note that the embryonic cells from WT mice show an oval shape and smooth edge, while the embryonic cells from KO mice show irregular shapes and rough edges. (B). The average numbers of total and fertilized oocytes collected from WT or KO female mice on dpc 1.5. (C). Representative images of oocytes ovulated by gonadotropin-treated WT and Kdm3bKO mice on dpc 1.5. The schedules for PMSG and hCG treatments of female mice are indicated. Fertile WT male mice were housed with treated females on day 3 . Ovulation was examined in the morning (dpc 1.5 ) on day 5. (D). The average numbers of total and fertilized oocytes collected from gonadotropin-treated WT or KO female mice on dpc 1.5. Data in panels B and D are presented as mean \pm standard deviation. *, $\mathrm{p}<0.05$ by Student's $t$ test. 
A
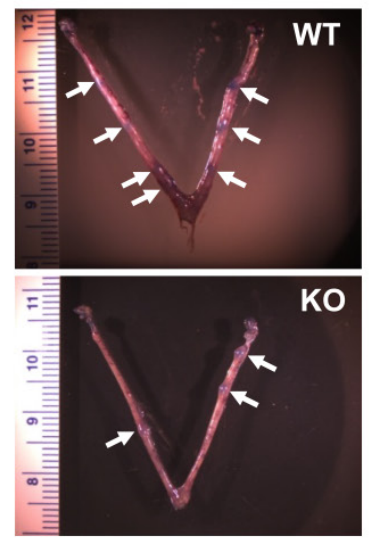

B

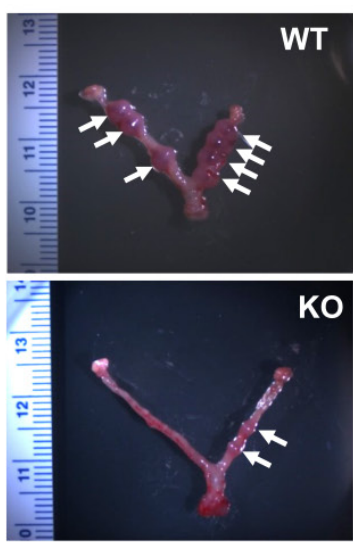

C

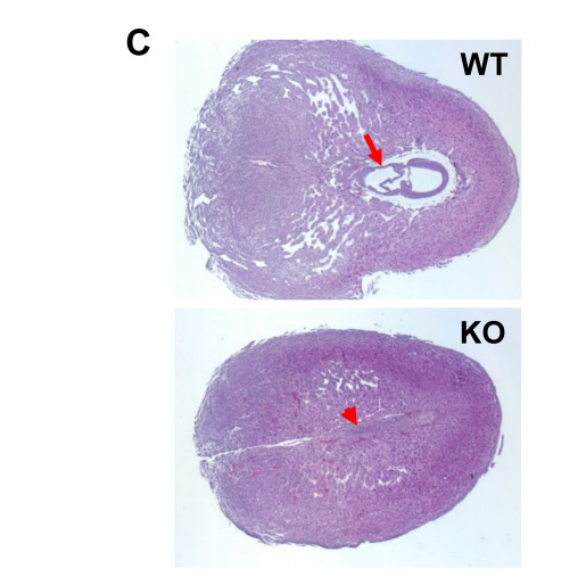

C
D

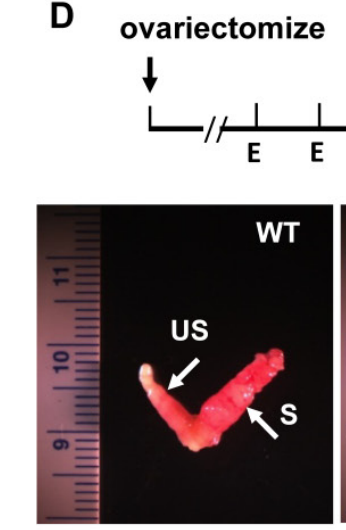

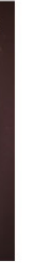

ko

\section{.}
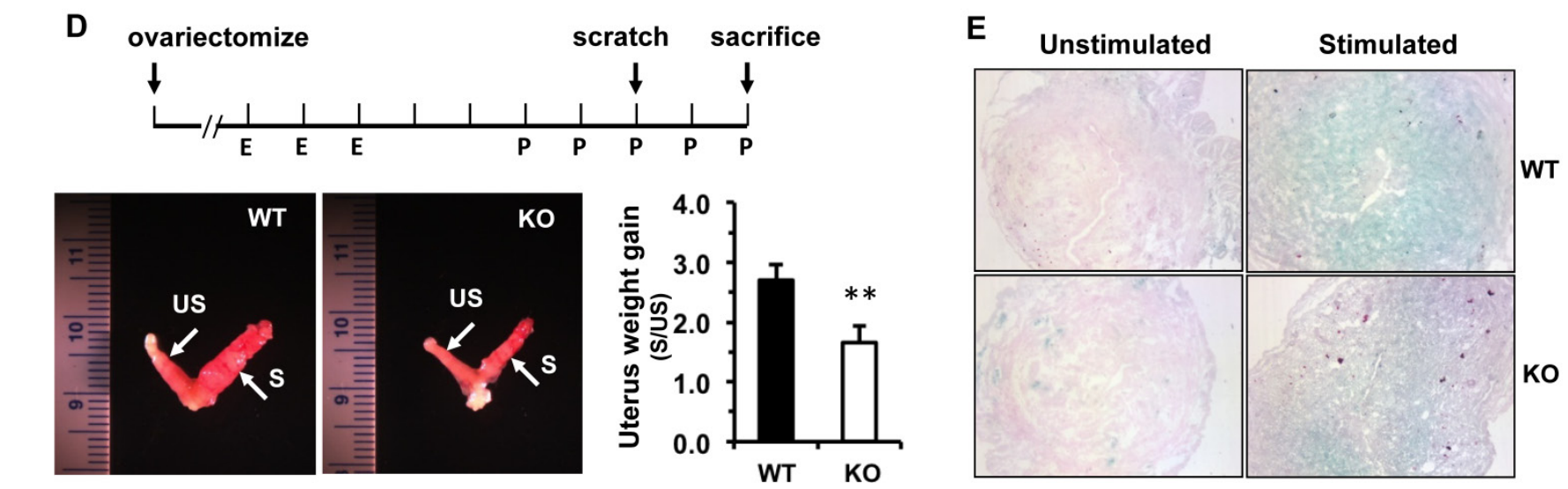

Figure 7. Kdm3b is required for normal decidualization. (A). Representative images of the reproductive tracts of the female WT and Kdm3bKO (KO) mice. Arrows indicate the embryo implantation sites stained by Chicago Blue dye in the uteri on dpc 4.5. (B). Representative images of the reproductive tracts of WT and KO mice on dpc 7.5. Arrows indicate larger and smaller embryos in the uteri of WT and KO mice, respectively. (C). The H\&E-stained images of cross sections prepared from pregnant uteri of WT and KO mice on dpc 7.5. The arrow indicates a normal embryo in WT uterus. The arrowhead indicates an implantation site in $\mathrm{KO}$ uterus where the embryo was absorbed. (D). Kdm3bKO mice exhibited a reduced decidual response. As sketched in the upper panel, ovariectomized WT mice $(n=8)$ and KO mice $(n=6)$ were treated with $17 \beta$-estradiol and progesterone. Decidulization was mechanically induced and measured as described in Materials and Methods. Representative images of uterine decidualization in WT and KO mice were shown. The ratios of the stimulated uterine horn (S) weights to un-stimulated uterine horn (US) weights were determined by weighing the wet uterine tissues. **, $\mathrm{p}<0.001$ by Student's $\mathrm{t}$ test. (E). Alkaline phosphotase activity detected by histochemistry (green color) in WT and KO uterine horns receiving decidual stimuli. The slides were counter-stained with Nuclear Fast Red.

A

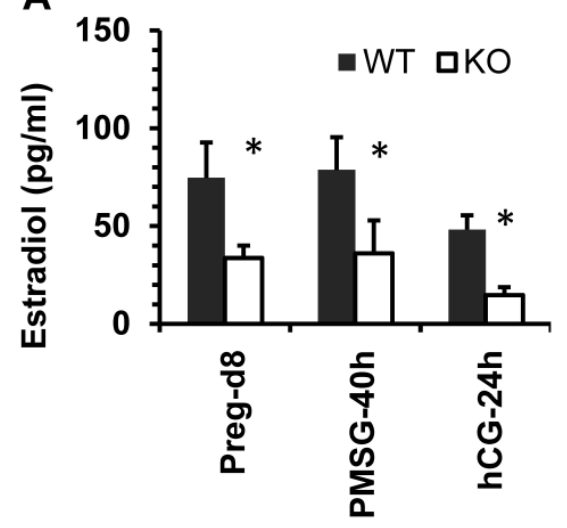

B

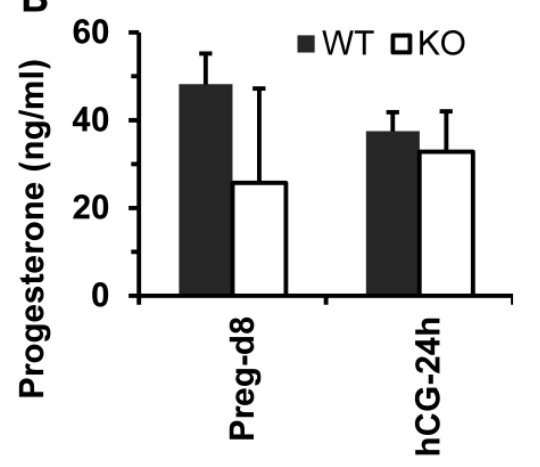

C

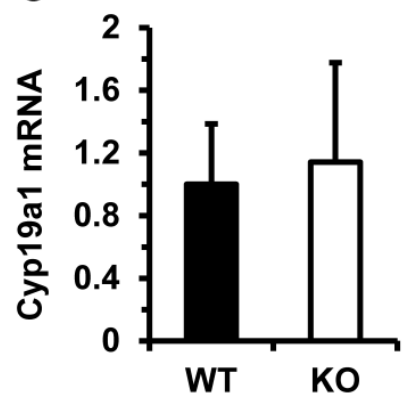

Figure 8. $\mathrm{Kdm} 3 \mathrm{~b}$ is required for maintaining normal levels of $17 \beta$-estradiol. $(\boldsymbol{A})$. The concentrations of $17 \beta$-estradiol in the serum samples prepared from WT and Kdm3bKO (KO) mice on day 8 of pregnancy $(n=6)$, at 40 hours after PMSG injection $(n=6)$, and at 24 hours after hCG injection $(n=3)$. (B). The concentrations of progesterone in the serum samples of WT and KO mice on day 8 pregnancy $(n=6)$ and at 24 hours after hCG injection $(n=3)$. (C). The relative expression levels of Cyp19al mRNA in the ovaries of untreated WT and KO mice $(n=5)$. 


\section{Discussion}

The pituitary-secreted GH stimulates IGF-1 expression in various tissues to regulate somatic growth. IGF-1 and IGFBP-3 form a stable protein complex in the circulating blood to maintain a relatively constant IGF-1 concentration, which is required for normal somatic growth and other physiological functions [24, 25]. The kidney possesses the highest expression level of IGFBP-3, which plays a key role to maintain IGF-1 stability in the circulation [19]. It has been shown that knockout of IGF-1 in mice reduces IGF-1 in the blood and decreases somatic growth [26-28], while overexpression of IGFBP-3 in transgenic mice increases IGF-1 in the blood [29]. We have previously shown that liver-specific overexpression of IGF-1 moderately enhances somatic growth and partially prevents the effects of GH deficiency [30]. Knockout of the nuclear receptor coactivator 3 (NCOA3, also known as SRC-3) decreases IGFBP-3 expression in the kidney, which in turn causes fast IGF-1 degradation in the blood and postnatal somatic growth retardation [19]. In the present study, we found that $\mathrm{Kdm} 3 \mathrm{bKO}$ mice exhibit postnatal growth retardation and this dwarf phenotype is kept through their lifespan. Mechanistic analysis revealed that IGF-1 mRNA expressed in the liver is unaffected, suggesting that GH secretion and GH-induced IGF-1 expression in the liver are independent of $\mathrm{Kdm} 3 \mathrm{~b}$ function. However, serum IGF-1 is low, and this is caused by accelerated degradation of IGF-1 in the circulation. Since we found that $\mathrm{Kdm} 3 \mathrm{~b}$ is highly expressed in the kidney, and IGFBP-3 mRNA and IGFBP-3 protein are significantly reduced in the kidney and sera of $\mathrm{Kdm} 3 \mathrm{bKO}$ mice, we propose that the decreased IGFBP-3 expression caused by $\mathrm{Kdm} 3 \mathrm{~b}$ deficiency is responsible for the decreased IGF-1 level in the blood, which may partially explain the growth retardation of $\mathrm{Kdm} 3 \mathrm{bKO}$ mice. It would be interesting to find out how $\mathrm{Kdm} 3 \mathrm{~b}$ exactly regulates the expression of IGFBP-3 in the kidney in a future study.

A successful reproductive process is a result of coordinated functions of multiple organs. In female, oocytes are developed in the follicles of ovaries and released into the fallopian tubes, where they meet the sperms and get fertilized [31]. Blastocysts derived from the fertilized oocytes enter the steroid hormone-primed uterine horns and attach to the uterine wall. This attachment induces uterine stromal cell proliferation and differentiation into the epitheloid-decidual cells, a process termed as decidualization [23]. Decidualization establishes the connection between mom and fetus and then, the placenta develops to nurture the embryos until birth. The coordinated stimuli of estrogen and progesterone and the appropriate responses of the female reproductive or- gans to these stimuli play essential roles in the regulation of female reproductive events, including estrous cycle, sexual behavior, oocyte maturation, ovulation, uterine receptivity, embryo implantation, uterine decidualization, pregnant maintenance and parturition [23, 32]. In the present study, we showed that $\mathrm{Kdm} 3 \mathrm{~b}$ is broadly expressed in the follicular and interstitial cells of the ovary, the luminal epithelial cells of the oviduct, the epithelial and stromal cells of the endometrium, and the smooth muscle cells of the myometrium. Kdm3bKO null mice exhibited normal ovarian and uterine morphogenesis, follicular development, estrogen-induced uterine growth and successful coitum, suggesting that $\mathrm{Kdm} 3 \mathrm{~b}$ function is not essential for these female organ morphogenesis and reproductive events. However, the female $\mathrm{Kdm} 3 \mathrm{bKO}$ mice are not able to produce any pups, indicating $\mathrm{Kdm} 3 \mathrm{~b}$ is absolutely required for completing the entire reproductive process. Analysis of the sequential reproductive events demonstrated that $\mathrm{Kdm} 3 \mathrm{bKO}$ mice exhibited prolonged estrous cycles, decreased ovulation capacity, and reduced fertilization rate. These knockout mice also showed significantly decreased embryo implantation sites in their uteri, which could be a consequence of the reduced ovulation and fertilization. There results demonstrate that $\mathrm{Kdm} 3 \mathrm{~b}$ is required for maintaining normal estrous cycle, ovulation capacity and fertilization efficiency.

Furthermore, we also found that the implanted embryos in $\mathrm{Kdm} 3 \mathrm{bKO}$ uteri were reduced in number and were much smaller than those embryos in WT uteri. Many of the implanted embryos in $\mathrm{Kdm} 3 \mathrm{bKO}$ uteri died before dpc 7.5. These defects were associated with the significantly reduced decidual response in these $\mathrm{Kdm} 3 \mathrm{bKO}$ female mice. Since appropriate decidualization of the endometrial stroma is essential for establishing the connection between embryo and uterine stroma as well as for placental development, the impaired decidual response may be responsible for the arrested growth and death of the implanted embryos.

Maintenance of an appropriate level of H3K9 methylation has been shown to play essential roles in mammalian reproduction. Suv39h1 and suv39h2, two of the H3K9 methyltransferases, are required for heterochromatin formation and genome stability in male germ cells [33]. G9a, another H3K9 methyltransferase, is required for maintaining $\mathrm{H} 3 \mathrm{~K} 9 \mathrm{me} 1$ and $\mathrm{H} 3 \mathrm{~K} 9 \mathrm{me} 2$ signals and synchronous synapsis in the meiotic prophase [34]. Our previous study has shown that Kdm3a demethylates H3K9 in the spermatocytes. Knockout of $\mathrm{Kdm} 3 \mathrm{a}$ increases $\mathrm{H} 3 \mathrm{~K} 9$ methylation and disturbs the expression of genes required for chromatin condensation and spermatid elongation and maturation, causing male infertility [14]. In the present 
study, we demonstrated that knockout of $K d m 3 b$ increases the levels of $\mathrm{H} 3 \mathrm{~K} 9 \mathrm{me} 1 / 2 / 3$ in the follicular and/or interstitial cells of the ovary and in the endometrial stroma and/or epithelial cells of the uterus. Since these changes of $\mathrm{H} 3 \mathrm{~K} 9 \mathrm{me} 1 / 2 / 3$ levels could be detected by IHC and Western blotting, the alterations of these histone codes caused by $K d m 3 b$ knockout must involve extensive chromatin regions. Therefore, these alterations should affect the expression of many genes. The altered gene expression profiles may serve as a molecular basis for the infertile phenotype of $\mathrm{Kdm} 3 \mathrm{bKO}$ mice. However, the direct target genes of $\mathrm{Kdm} 3 \mathrm{~b}$ in the ovary and uterus are currently unclear. Since knockout of $K d m 3 b$ affects global H3K9me1/2/3 levels in these organs, it would be difficult to identify the specific target genes and pathways responsible for the impaired reproductive functions of female $\mathrm{Kdm} 3 \mathrm{bKO}$ mice. This should warrant additional studies to map the Kdm3b-regulated genes and their underlying molecular mechanisms that control female reproductive function.

In summary, we have identified $\mathrm{Kdm} 3 \mathrm{~b}$ as a key H3K9 demethylase essential for postnatal somatic growth and female reproductive function. Disruption of $\mathrm{Kdm} 3 \mathrm{~b}$ function decreases IGFBP-3 expression, which results in fast degradation of IGF-1 and small body size. The loss of $\mathrm{Kdm} 3 \mathrm{~b}$ function also prolongs female estrous cycle, and decreases ovulation capacity, oocyte fertilization rate, embryo implantation, decidual response and embryo growth. Together, these defects in reproductive function result in a female infertile phenotype. These defects are associated with extensive alterations of $\mathrm{H} 3 \mathrm{~K} 9 \mathrm{me} 1, \mathrm{H} 3 \mathrm{~K} 9 \mathrm{me} 2$ and/or $\mathrm{H} 3 \mathrm{~K} 9 \mathrm{me} 3$ levels in the ovarian and uterine cells where $\mathrm{Kdm} 3$ is highly expressed. These findings suggest that $K D M 3 B$ mutation or disrupted pathways regulated by KDM3B might be present in human patients with growth retardation and/or female reproductive problems.

\section{Supplementary Material}

Figures S1-S2, Table S1.

http://www.ijbs.com/v11p0494s1.pdf

\section{Abbreviations}

BSA, bovine serum albumin; dpc, day(s) post cuitum; ES, embryonic stem; GH, growth hormone; $\mathrm{H} \& \mathrm{E}$ staining, hematoxylin and eosin staining; hCG, human chorionic gonadotropin; HRP, horseradish peroxidase; IGF-1, insulin-like growth factor 1; IGFBP-3, insulin-like growth factor binding protein-3; IHC, immunohistochemistry; Kdm3b $\%$, Kdm3b knockout (Kdm3KO); PMSG, pregnant mare's serum gonadotropin; SDS-PAGE, sodium dodecyl sulfate polyacrylamid gel electrophoresis; WT, wild type.

\section{Acknowledgements}

We thank the BCM Transgenic Mouse Core for microinjection of ES cells, and this core is partially supported by a cancer center grant (P30-CA125123) from National Institutes of Health. This study was supported by National Institutes of Health grants DK058242, CA112403 and DK059820 and a Cancer Prevention and Research Institute of Texas grant RP120732-P5 to J.X.

\section{Competing Interests}

The authors have declared that no competing interest exists.

\section{References}

1. Kouzarides T. Chromatin modifications and their function. Cell. 2007; 128: 693-705.

2. Bernstein BE, Kamal M, Lindblad-Toh K, et al. Genomic maps and comparative analysis of histone modifications in human and mouse. Cell. 2005; 120: 169-81.

3. Roh TY, Cuddapah S, Cui K, et al. The genomic landscape of histone modifications in human T cells. Proc Natl Acad Sci U S A. 2006; 103: 15782-7.

4. Barski A, Cuddapah S, Cui $\mathrm{K}$, et al. High-resolution profiling of histone methylations in the human genome. Cell. 2007; 129: 823-37.

5. Heintzman ND, Stuart RK, Hon G, et al. Distinct and predictive chromatin signatures of transcriptional promoters and enhancers in the human genome. Nat Genet. 2007; 39: 311-8.

6. Lim S, Metzger E, Schule R, et al. Epigenetic regulation of cancer growth by histone demethylases. Int J Cancer. 2010; 127: 1991-8.

7. Kim JY, Kim KB, Eom GH, et al. KDM3B is the H3K9 demethylase involved in transcriptional activation of lmo2 in leukemia. Mol Cell Biol. 2012; 32: 2917-33.

8. Brauchle M, Yao Z, Arora R, et al. Protein complex interactor analysis and differential activity of KDM3 subfamily members towards H3K9 methylation. PLoS One. 2013; 8: e60549.

9. Krieg AJ, Rankin EB, Chan D, et al. Regulation of the histone demethylase JMJD1A by hypoxia-inducible factor 1 alpha enhances hypoxic gene expression and tumor growth. Mol Cell Biol. 2010; 30: 344-53.

10. $\mathrm{Hu} \mathrm{Z}$, Gomes I, Horrigan SK, et al. A novel nuclear protein, 5qNCA (LOC51780) is a candidate for the myeloid leukemia tumor suppressor gene on chromosome 5 band q31. Oncogene. 2001; 20: 6946-54

11. Wolf SS, Patchev VK, Obendorf M. A novel variant of the putative demethylase gene, s-JMJD1C, is a coactivator of the AR. Arch Biochem Biophys. 2007; 460: 56-66

12. Lai F, Godley LA, Fernald AA, et al. cDNA cloning and genomic structure of three genes localized to human chromosome band $5 \mathrm{q} 31$ encoding potential nuclear proteins. Genomics. 2000; 70: 123-30.

13. Paolicchi E, Crea F, Farrar WL, et al. Histone lysine demethylases in breast cancer. Crit Rev Oncol Hematol. 2013; 86: 97-103.

14. Liu Z Zhou S, Liao L, et al. Jmjd1a demethylase-regulated histone modification is essential for cAMP-response element modulator-regulated gene expression and spermatogenesis. J Biol Chem. 2010; 285: 2758-70.

15. Okada Y, Scott G, Ray MK, et al. Histone demethylase JHDM2A is critical for Tnp1 and Prm1 transcription and spermatogenesis. Nature. 2007; 450: 119-23.

16. Tateishi K, Okada Y, Kallin EM, et al. Role of Jhdm2a in regulating metabolic gene expression and obesity resistance. Nature. 2009; 458: 757-61.

17. Deng C, Wynshaw-Boris A, Zhou F, et al. Fibroblast growth factor receptor 3 is a negative regulator of bone growth. Cell. 1996; 84: 911-21.

18. Liu W, Selever J, Wang D, et al. Bmp4 signaling is required for outflow-tract septation and branchial-arch artery remodeling. Proc Natl Acad Sci U S A. 2004; 101: 4489-94.

19. Liao L, Chen X, Wang S, et al. Steroid receptor coactivator 3 maintains circulating insulin-like growth factor I (IGF-I) by controlling IGF-binding protein 3 expression. Mol Cell Biol. 2008; 28: 2460-9.

20. Kawagoe J, Li Q, Mussi P, et al. Nuclear receptor coactivator-6 attenuates uterine estrogen sensitivity to permit embryo implantation. Dev Cell. 2012; 23: 858-65.

21. Xu J, Qiu Y, DeMayo FJ, et al. Partial hormone resistance in mice with disruption of the steroid receptor coactivator-1 (SRC-1) gene. Science. 1998; 279: 1922-5.

22. Jeong JW, Lee HS, Lee KY, et al. Mig-6 modulates uterine steroid hormone responsiveness and exhibits altered expression in endometrial disease. Proc Natl Acad Sci U S A. 2009; 106: 8677-82.

23. Wang H, Dey SK. Roadmap to embryo implantation: clues from mouse models. Nat Rev Genet. 2006; 7: 185-99. 
24. Stewart CE, Rotwein P. Growth, differentiation, and survival: multiple physiological functions for insulin-like growth factors. Physiol Rev. 1996; 76: 1005-26.

25. Jones JI, Clemmons DR. Insulin-like growth factors and their binding proteins: biological actions. Endocr Rev. 1995; 16: 3-34.

26. Liu JP, Baker J, Perkins AS, et al. Mice carrying null mutations of the genes encoding insulin-like growth factor I (Igf-1) and type 1 IGF receptor (Igf1r). Cell. 1993; 75: 59-72.

27. Powell-Braxton L, Hollingshead P, Warburton C, et al. IGF-I is required for normal embryonic growth in mice. Genes Dev. 1993; 7: 2609-17.

28. Ueki I, Ooi GT, Tremblay ML, et al. Inactivation of the acid labile subunit gene in mice results in mild retardation of postnatal growth despite profound disruptions in the circulating insulin-like growth factor system. Proc Natl Acad Sci U S A. 2000; 97: 6868-73.

29. Modric T, Silha JV, Shi Z, et al. Phenotypic manifestations of insulin-like growth factor-binding protein-3 overexpression in transgenic mice. Endocrinology. 2001; 142: 1958-67.

30. Liao L, Dearth RK, Zhou S, et al. Liver-specific overexpression of the insulin-like growth factor-I enhances somatic growth and partially prevents the effects of growth hormone deficiency. Endocrinology. 2006; 147: 3877-88.

31. Kolibianakis EM, Papanikolaou EG, Fatemi HM, et al. Estrogen and folliculogenesis: is one necessary for the other? Curr Opin Obstet Gynecol. 2005; 17: 249-53.

32. Lee KY, Jeong JW, Tsai SY, et al. Mouse models of implantation. Trends Endocrinol Metab. 2007; 18: 234-9.

33. Peters AH, O'Carroll D, Scherthan H, et al. Loss of the Suv39h histone methyltransferases impairs mammalian heterochromatin and genome stability. Cell. 2001; 107: 323-37.

34. Tachibana M, Nozaki M, Takeda N, et al. Functional dynamics of H3K9 methylation during meiotic prophase progression. EMBO J. 2007; 26: 3346-59. 